\title{
STUDY OF GASEOUS PHASE IN DIAMONDS WITH ECLOGITIC AND ULTRABASIC INCLUSIONS FROM YAKUTIAN KIMBERLITE PIPES.
}

\section{S.B. Tal'nikova; Yu. P. Barashkov and I.M. Svoren'}

Yakutsk Institute of Geosciences (USSR).

The reproducibility of the results obtained by various degassing methods for diamonds from different deposits suggests that the liberated gases are relicts of a fluid of the diamond crystallization medium.

To get additional information and to test a possible relationship of gas composition of diamonds to their inclusions paragenesis and to the presence of solid inclusions, we studied diamonds whose paragenesis was in most cases known from the composition of solid inclusions.

Gases which are sorbed in dislocations, vacansies, microcracks and other lattice defects of diamond were liberated by neating a sample in an inert atmosphere using special device connected with an MX-1303 mass-spectrometer. Diamond cristals where thoroughly cleaned immediately prior to analysis using a specially developed scheme. Before each analysis, the device was degassed by heating to $423 \ldots . . .473 \mathrm{~K}$. Cooling was performed at a constant evacuation value $\left(10^{-7}\right.$ Torr). The cleaned crystal was placed in a glass capsule. After the device was mounted and connected with mass-spectrometer, the system was evacuated up to $1 * 10^{-7}$ Torr. Due to minimum weight of the sample, heating was carried out at $T=693$ and $1593 \mathrm{~K}$. The gases thus obtained were fed to the mass-spectrometer.

We analized six diamond crystals from Udachnaya pipe (samples 3165, 3027, 3038, 3689, 3207, 3037) one (sample 1169) from Mir and one (sample 2086) from Aykhal. Crystals 3689, 3207, 2086, and 1169 were colourless, flat-faceted octahedra, 3027 was a 
yellow cube, 3038 was a grey opaque cube, 3037 was a macle of a coated diamond, and 3165 was a crystal of indefinite shape. Octahedral crystals 3689 and 1169 contained olivine and pyrope-almandine inclusions respectively. These crystals were sawn in such a manner that one half contained inclusions and the other nad no visible inclusions. Crystal 3037 was preliminarily crushed and its yellow coat separated from the transparent core. The studied samples varied in weight from 4.4 to $194.3 \mathrm{mg}$. The concentration and composition of liberated gases vary from crystal to crystal. All samles contain $\mathrm{H}_{2}, \mathrm{~N}_{2}$ and $\mathrm{CO}_{2}$. Other gases are present in only some of the crystals: $C O$ in 1169,3689 , $3037 ; \mathrm{H}_{2} \mathrm{O}$ in $2086,3165,3038,1169,3207,3037 ; \mathrm{CH}_{4}$ in 3038 , 1169, 3207, 3037; $\mathrm{C}_{2} \mathrm{H}_{6}$ in 3038. Both halves of 3689 have gases of similar composition but the half with olivine inclusions is somewhat higher in $\mathrm{CO}_{2}, \mathrm{CO}$ and $\mathrm{N}_{2}$. The situation is different in crystal 1169. $\mathrm{N}_{2}, \mathrm{CO}_{2}$ and $\mathrm{CH}_{4}$ contents are several times higher in the half with pyrope-almandines compared to the half free of visible inclusions. The latter half also contains $c 0$ and $\mathrm{H}_{2} \mathrm{O}$. In 3037, gas content of the transparent core is much higher than that of the yellow coat. Gases in the grey cube 3038 differ in composition not only from the yellow cube 3027 but also from the rest of the studied samples. It was the only crystal which contained the $\mathrm{C}_{2} \mathrm{H}_{6}$ homologue. The yeild of gases ( in $8 / 8$ of crystal) varies widely, from $47.699 * 10^{-6}$ to $684.974 * 10^{-6}$, in eclogitic diamonds and in a narrower interval, from $4.221 * 10^{-6}$ to $5.247 * 10^{-6}$, in ultrabasic diamonds.

This, the present study has revealed that gas composition is dependent on the presence of solid inclusions in diamond. Higher gas cohtents of the diamond halves containing mineral inclusions are probably due to sorbtion of volatiles at the diamond-inclusion interface. Gas contents of eclogitic diamonds are an order of magnitude higher than those of ultrabasic diamonas. 